\title{
THE TRIANGULABILITY OF THE ORBIT SPACE OF A DIFFERENTIABLE TRANSFORMATION GROUP'
}

\author{
BY C. T. YANG
}

\section{Communicated by Deane Montgomery, December 13, 1962}

The purpose of this note is to prove the result as given in the title. The result was suggested to the author by Professor D. Montgomery to whom the author wishes to express his warmest thanks.

By a differentiable manifold we mean a locally euclidean separable metric space on which there is a differentiable structure. It may not be connected and its components may not be of the same dimension. If $M$ is a differentiable manifold and $G$ is a compact Lie group which acts differentiably on $M$, we call the pair $(G, M)$ a differentiable transformation group.

Let $(G, M)$ be a differentiable transformation group. Whenever $x \in M, G_{x}$ denotes the isotropy group at $x$. Whenever $H$ is a closed subgroup of $G,(H)$ denotes the conjugate class of $H$ in $G$ and $M_{(H)}$ denotes the set $\left\{x \in M \mid G_{x} \in(H)\right\}$. It is clear that $G M_{(H)}=M_{(H)}$. If $A$ is a subset of $M$ with $G A=A$, the space of the orbits in $A$ is denoted by $A / G$. The natural projection of $M$ onto $M / G$ is denoted by $p$.

LeMma 1. Whenever $H$ is a closed subgroup of $G, M_{(H)}$ is a differentiable submanifold of $M$ and $p$ defines a fibring map $p_{(H)}: M_{(H)}$ $\rightarrow M_{(H)} / G$. Hence $M_{(H)} / G$ can be made a differentiable manifold such that $p_{(H)}$ is differentiable.

PRoof. Let $x$ be an arbitrary point of $M_{(H)}$. Since $G$ acts differentiably on $M$, there is a slice $S$ at $x$ which is a differentiable open cell in $M$ and on which there is an admissible coordinate system with respect to which $G_{x}$ acts orthogonally [1]. Denote by $F$ the stationary point set of $G_{x}$ in $S$. Then $F$ is a differentiable open cell in $M$ and $G F$ is a neighborhood of $x$ in $M_{(H)}$. Since the map of $G / G_{x} \times F$ into $G F$ given by $\left(g G_{x}, y\right) \rightarrow g y$ is a homeomorphism onto, it follows that $G F$ is a differentiable submanifold of $M$. Hence $M_{(H)}$ is a differentiable submanifold of $M$. Moreover, $p_{(H)}$ is a fibring map with $F$ being a differentiable local cross-section at $x$. Hence $M_{(H)} / G$ can be made a differentiable manifold such that $p_{(H)}$ is differentiable. The proof of Lemma 1 is thus completed.

By a differentiable $n$-space, $n=0,1, \cdots$, we mean a pair $(X, \Sigma)$

1 The research is sponsored by the U.S. Army Research Office (Durham) under Grant CRD-AA-M-2056. 
which consists of an $n$-dimensional separable metric space $X$ and a partition $\Sigma$ of $X$ satisfying the following conditions:

(a) Every $\sigma \in \Sigma$ is a connected differentiable manifold.

(b) $\Sigma$ is locally finite.

(c) Given any $x \in \sigma_{0} \in \Sigma$ with $\operatorname{dim} \sigma_{0}=r$, there is a neighborhood $U$ of $x$ in $X$ and a differentiable $k$-space $\left(X^{\prime}, \Sigma^{\prime}\right)$ with $k<n-r$ such that (i) $\sigma^{\prime} \in \Sigma^{\prime}$ if and only if $\sigma^{\prime}=X^{\prime} \cap \sigma$ for some $\sigma \in \Sigma$ with $x \in \bar{\sigma}-\sigma$ and (ii) there is a homeomorphism of $\vec{U}$ onto the join $S^{r} * X^{\prime}$ of a closed $r$-simplex $S^{r}$ and $X^{\prime}$ which maps $U \cap \sigma_{0}$ diffeomorphically onto the interior of $S^{r}$ and which maps, for every $\sigma \in \Sigma$ with $x \in \bar{\sigma}-\sigma, U \cap \sigma$ diffeomorphically onto $S^{r} *\left(X^{\prime} \cap \sigma\right)-\left(S^{r} \cup\left(X^{\prime} \cap \sigma\right)\right)$, where $S^{r} *\left(X^{\prime} \cap \sigma\right)$ is the join of $S^{r}$ and $X^{\prime} \cap \sigma$.

Notice that, if $n=0$, then, by (a) and (b), $X$ is a countable discrete space and $\Sigma=X$ so that (c) is trivial. Hence, by recursion, differentiable $n$-spaces are well-defined for all non-negative integers $n$.

Let $\sigma$ and $\tau$ be two distinct members of $\Sigma$ with $\sigma \cap \bar{\tau} \neq \varnothing \varnothing$. It follows from (c) that every $x \in \sigma \cap \bar{\tau}$ has a neighborhood $U$ with $U \cap \sigma \subset \bar{\tau}$ and that every $x \notin \sigma-\bar{\tau}$ has a neighborhood not meeting $\bar{\tau}$. Therefore $\sigma \cap \bar{\tau}$ is both open and closed in $\sigma$ and hence, by the connectedness of $\sigma, \sigma \subset \bar{\tau}$. Let $x \in \sigma \cap \bar{\tau}$ and let $U$ be a neighborhood of $x$ as described in (c). Then $U \cap \tau$ is homeomorphic to $(U \cap \sigma) *\left(X^{\prime} \cap \tau\right)$ $-\left((U \cap \sigma) \cup\left(X^{\prime} \cap \tau\right)\right)$. Hence $\operatorname{dim} \sigma<\operatorname{dim} \tau$. This proves

(d) Whenever $\sigma$ and $\tau$ are two distinct members of $\Sigma$ with $\sigma \cap \bar{\tau}$ $\neq \varnothing, \sigma \subset \bar{\tau}$ and $\operatorname{dim} \sigma<\operatorname{dim} \tau$.

Lemma 2. Differentiable n-spaces are regular locally polyhedral spaces in the sense of Cairns [2] and hence are triangulable.

Proof. In [2] one may find the definition of a regular locally polyhedral space and the theorem that regular locally polyhedral spaces are triangulable.

We have seen that differentiable 0 -space are countable discrete spaces so that they are trivial regular locally polyhedral spaces. Hence we may proceed by induction on $n$ and assume that differentiable $k$-spaces with $k<n$ are regular locally polyhedral spaces.

Let $(X, \Sigma)$ be a differentiable $n$-space and let $x$ be any point of $X$. By (c), there is a differentiable $k$-space $\left(X^{\prime}, \Sigma^{\prime}\right)$ with $k<n$ and a neighborhood $U$ of $x$ in $X$ such that $\bar{U}$ is homeomorphic to the join of a closed simplex and $X^{\prime}$. Since, by induction hypothesis, $X^{\prime}$ is a regular locally polyhedral space so that it is triangulable, $\bar{U}$ is triangulable and thus $U$ is homeomorphic to an open subset of a polyhedron. Hence $X$ is locally polyhedral. 
Now, as one may see,

$$
X=\bigcup_{\sigma \in \Sigma} \sigma
$$

is a general locally polyhedral space in the sense of [2]. To complete the proof, it remains to be shown that any distinct $\sigma_{0}, \tau \in \Sigma$ with $\sigma_{0} \subset \bar{\tau}$ are differentiably related in the sense of [2]. By (c), there is a neighborhood $U$ of $x$ and a differentiable $k$-space $\left(X^{\prime}, \Sigma^{\prime}\right)$ with $k<n$ such that (i) $\sigma^{\prime} \in \Sigma^{\prime}$ if and only if $\sigma^{\prime}=X^{\prime} \cap \sigma$ for some $\sigma \in \Sigma$ with $x \in \bar{\sigma}-\sigma$ and (ii) there is a homeomorphism $h$ of $\bar{U}$ onto $S * X^{\prime}$ for some closed simplex $S$ which maps $U \cap \sigma_{0}$ diffeomorphically on to the interior of $S$ and which maps, for every $\sigma \in \Sigma$ with $x \in \bar{\sigma}-\sigma, U \cap \sigma$ diffeomorphically onto $S *\left(X^{\prime} \cap \sigma\right)-\left(S \cup\left(X^{\prime} \cap \sigma\right)\right)$. By induction hypothesis, $\left(X^{\prime}, \Sigma^{\prime}\right)$ is a regular locally polyhedral space so that for every $\sigma \in \Sigma$ with $X^{\prime} \cap \sigma \subset X^{\prime} \cap(\bar{\tau}-\tau), X^{\prime} \cap \sigma$ and $X^{\prime} \cap \tau$ are differentiably related. Therefore for every $y \in X^{\prime} \cap \bar{\tau}$, there is a coordinate system whose domain $D$ is a closed cell in $X^{\prime} \cap \bar{\tau}$ containing $y$. That means, there is a homeomorphism $h^{\prime}$ of $D$ onto a closed simplex $S^{\prime}$ such that if $\sigma$ is a member of $\Sigma$ with $D \cap \sigma \neq \varnothing$, the interior $I^{\prime}(D \cap \sigma)$ of $D \cap \sigma$ in $X^{\prime} \cap \sigma$ is not empty and $h^{\prime}$ maps $I^{\prime}(D \cap \sigma)$ diffeomorphically onto the interior of a face of $S^{\prime}$. Let $h^{\prime \prime}$ be the natural homeomorphism of $S * D$ onto $S_{1}=S * S^{\prime}$ induced by $h^{\prime}$. Then $h_{1}=h^{\prime \prime} h$ is a homeomorphism of $D_{1}=h^{-1}(S * D)$ onto $S_{1}$. Since $S_{1}$ is a closed simplex of the same dimension as $\bar{\tau}$, it gives a coordinate system with domain $D_{1}$. Moreover, it is easily seen that if $\sigma$ is a member of $\Sigma$ with $D_{1} \cap \sigma \neq \varnothing$, then the interior $I\left(D_{1} \cap \sigma\right)$ of $D_{1} \cap \sigma$ in $\sigma$ is not empty and $h_{1}$ maps $I\left(D_{1} \cap \sigma\right)$ diffeomorphically onto the interior of a face of $S_{1}$. Hence $U \cap \sigma_{0}$ and $U \cap \tau$ are differentiably related. But $U$ is a neighborhood of an arbitrary point of $\sigma_{0}$. We infer that $\sigma_{0}$ and $\tau$ are differentiably related. The proof of Lemma 2 is thus completed.

Now we are ready to give our main result.

Theorem. Let $M$ be a differentiable manifold and let $G$ be a compact Lie group acting differentiably on $M$. Then the orbit space $M / G$ is triangulable.

Proof. Whenever $C$ is a component of $M, G C$ is finite-dimensional and is both open and closed in $M$. If we are able to prove $G C / G$ triangulable for every component $C$ of $M$, our theorem follows. Hence we may assume $M$ finite-dimensional. Under this assumption, $M / G$ is a finite-dimensional separable metric space. 
Whenever $H$ is a closed subgroup of $G,(H)$ denotes the conjugate class of $H$ in $G$ and

$$
M_{(H)}=\left\{x \in M \mid G_{x} \in(H)\right\} .
$$

As seen in Lemma 1, each component of $M_{(H)} / G$ is a connected differentiable manifold. Let $\Sigma$ be the set of all such manifolds. Then $\Sigma$ is clearly a partition of $M / G$. Hence, because of Lemma 2 , it remains to be shown that $(M / G, \Sigma)$ is a differentiable $n$-space, where $n=\operatorname{dim} M / G$.

The condition (a) is obvious by the construction of $\Sigma$. Let $x^{*} \in M / G$. Let $x \in p^{-1} x^{*}$ and let $S$ be a slice at $x$ which is a differentiable open cell in $M$ and on which there is an admissible coordinate system with respect to which $G_{x}$ acts orthogonally. It is known [3] that $S$ meets only finitely many $M_{(H)}$. Since for every $(H),\left(G S \cap M_{(H)}\right) / G$ is connected, it follows that $G S / G$ is a neighborhood of $x^{*}$ which meets only finitely many members of $\Sigma$. Hence the condition (b) is proved.

To prove the condition (c), we apply induction on $n=\operatorname{dim} \cdot M / G$. Let $x^{*}, x, S$ be as above and let $S^{\prime}$ be a spherical neighborhood of $x$ in $S$ with $\bar{S}^{\prime} \subset S$. Since $G_{x}$ acts orthogonally, the stationary point set $F$ of $G_{x}$ in $\bar{S}^{\prime}$ is a linear closed cell. Let $Y$ be the boundary of the linear closed cell $E$ which is the orthogonal complement of $F$ in $\bar{S}^{\prime}$. Then $Y$ is a sphere and $G_{x}$ acts orthogonally on $Y$. Morover, $\operatorname{dim} Y / G_{x}$ $<n$. Let $\Sigma^{\prime}$ be the partition for $Y / G_{x}$ as $\Sigma$ for $M / G$. Then, by induction hypothesis, $\left(Y / G_{x}, \Sigma^{\prime}\right)$ is a differentiable $k$-space for some $k<n$. Let $C$ be the convex shell of $\left(F \cap S^{\prime}\right) \cup\left(E \cap S^{\prime}\right)$ and let $U=G C / G$. Since there is a natural identification of $Y / G_{x}$ with $G Y / G$ and since $G_{x}$ acts orthogonally on $S$, one can easily verify that $U$ and $\left(Y / G_{x}, \Sigma^{\prime}\right)$ have the properties required in (c). Hence the proof is completed.

\section{BiBLIOGRAPHY}

1. D. Montgomery, H. Samelson and C. T. Yang, Exceptional orbits of highest dimension, Ann. of Math. (2) 64 (1956), 131-141.

2. S. S. Cairns, Triangulated manifolds and differentiable manifolds, Lectures in Topology, University of Michigan Press, Ann Arbor, Mich., 1941, pp. 143-157.

3. C. T. Yang, On a problem of Montgomery, Proc. Amer. Math. Soc. 8 (1957), 255-257.

University of Pennsylvania 